\title{
Comparison of Sliding Mode Controller for Classical and Direct Driven Electrohydraulic System
}

\author{
Juraj Benić, Željko Šitum, Mihael Cipek, Danijel Pavković, Josip Kasać \\ Department of Robotics and Production System Automation, Faculty of Mechanical Engineering and Naval Architecture \\ University of Zagreb, Zagreb HR-10000, Croatia \\ E-mail: jbenic@fsb.hr, zsitum@fsb.hr, mcipek@fsb.hr,dpavkovic@fsb.hr, jkasac@fsb.hr
}

\begin{abstract}
In this paper, a novel energy-efficient Direct Driven Hydraulic (DDH) drive is made in parallel with a classical valve-controlled proportional electrohydraulic system. In the proposed concept asymmetrical hydraulic cylinder is controlled with two reversible pumps directly connected to a servo motor. Due to direct control of the oil flow, such system provides higher energy efficiency in comparison to a valvecontrolled hydraulic system. The experimental setup is designed with the possibility of easy switching between both systems, which enables an exact comparison of the experimental results. Sliding Mode Controller (SMC) is designed for the DDH system and also for the classical proportional electro-hydraulic system. A comparison study of two systems is done based on the experimental results obtained with the SMC while reference results are obtained with a widely used PID controller. Parameters for the PID controller are obtained with the Ziegler-Nichols method. The same parameters are used for both controllers in all test cases. The performance and energy efficiency of the proposed system is evaluated through a step and sine wave reference signal with different payloads varying from 0 to $200 \mathrm{~kg}$ with $20 \mathrm{~kg}$ increments. Additionally, system performance is evaluated based on six parameters calculated from the system response while energy efficiency is calculated based on input and output powers of the systems.
\end{abstract}

Keywords: direct driven hydraulics, classical hydraulics, sliding mode control, system dynamics, energy efficiency

\section{Introduction}

Today, despite lower energy efficiency in comparison to electromechanical actuators, electrohydraulic systems due to their high power-to-weight ratio, self-cooling, fast response and lower cost are widely used in many applications such as non-road mobile machinery, manufacturing industry, robotics and aircrafts. Traditionally, valve-controlled systems that are energy-inefficient due to fluid throttling are used in such applications [1]. Disadvantages of the valve-controlled systems are overcome by displacement-controlled hydraulic systems or pump-controlled electrohydraulic systems [2, 3, 4]. In comparison to valve-controlled systems, servo-driven pump-controlled systems have compact structure, higher efficiency and the advantage of speed regulation loop [5].

Novel Direct Driven Hydraulic (DDH) drive is a new concept of a cylinder pump-controlled system firstly introduced in [6] and detailed described in [7]. In [8], a compact design of a DDH drive was investigated where the oil tank was replaced with an accumulator. It was shown that the larger pressure accumulator can replace the oil tank while the smaller one compensates for the pumps displacement error due to manufacturing. The energy efficiency of the proposed system was investigated in [9]. The tests showed that lifting efficiency varies from 48 to $20 \%$ while lowering efficiency varies from 32 to $8 \%$. Simulation results from the aspect of the energy efficiency are shown in [10] for the micro-excavator with three DDH units. Results showed that energy consumption due to the extra weight of the three DDH is 15\% higher, but approximately $20 \%$ more regeneration energy is produced. In [11] comparison of energy efficiency of a 5-ton excavator with a load sensing system was compared to three 
DDH units for front attachment. The simulation results showed that the overall energy efficiency of three DDH units is approximately $73.3 \%$ in comparison to $31.4 \%$ for the load sensing system. The influence of different types of oil on system efficiency is presented in [12]. It is shown that different types of oil can significantly increase system efficiency by almost $10 \%$ for temperatures below $-10^{\circ} \mathrm{C}$. From the aspect of the control theory in [13], a self-tuning fuzzy PID controller is used for controlling the DDH unit with the ability of online tuning making this controller more adaptive and effective in the proposed application. From the given literature it's concluded that the system dynamics between traditionally valve-controlled and DDH drive hasn't been compared yet.

In this paper, the DDH system is made in parallel with the classical proportional electrohydraulic system. An extensive analysis of the system dynamics and energy efficiency between DDH and classical system is presented. Due to nonlinear system dynamics, unknown and varying system parameters Sliding Mode Controller (SMC) is utilized for tracking the desired trajectory and stabilization of the system in a given set point. Both systems are subject to the same loads and working conditions allowing us a mutual comparison of the obtained results. Reference results are obtained with PID controller, while results obtained with an SMC are analyzed and compared to the reference results.

The rest of this paper is organized as follows. In Section 2 description of the experimental setup with used components and simplified schematic representation of the system is given. Section 3 describes used control algorithms while in Section 4 extensive analysis of the system dynamics is presented base on experimental results. In Section 5 energy efficiency is calculated based on power flow and compared. Conclusion and further work are given in Section 6.

\section{Experimental setup}

The experimental setup used in this research and a simplified schematics representation of the setup are shown in fig. 1. On the proposed setup, the classical valve-controlled proportional electro-hydraulic system and the DDH system are tested independently but under the same working conditions. The classical system uses a $1.1 \mathrm{~kW}$ AC motor with $1380 \mathrm{rev} / \mathrm{min}$ coupled with a $3.7 \mathrm{~cm}^{3} / \mathrm{rev}$ gear pump for ensuring constant pressure of a $60 \mathrm{bar}$. Proportional valve PRM2 from Argo-Hytos is used for motion control of a double-acting asymmetrical hydraulic cylinder 32/22x300 mm.

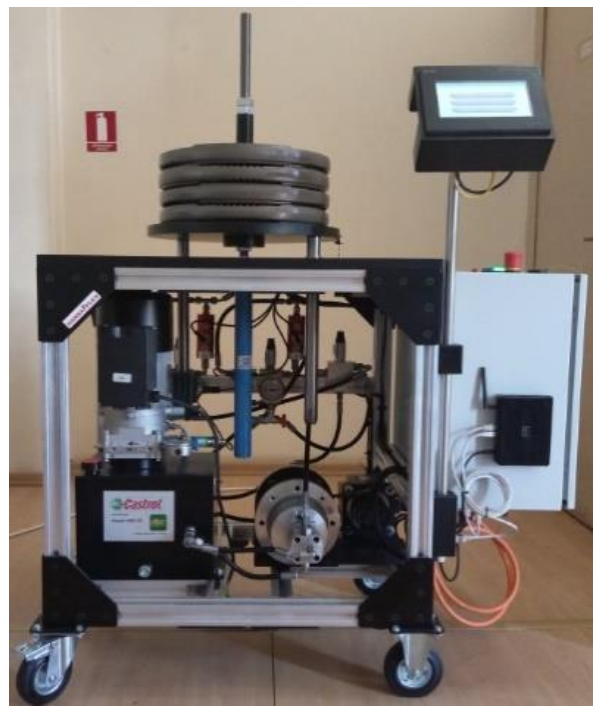

a)

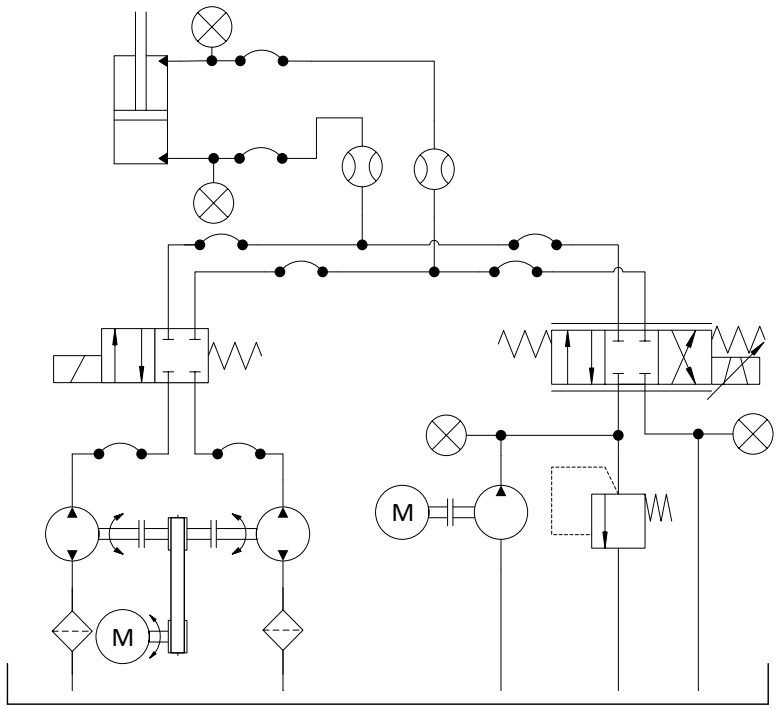

b)

Figure 1: Experimental setup - a) front view, b) schematics representation.

The DDH drive is shown in fig. 2. It uses two reversible Argo-Hytos gear pumps with a displacement of 4.8 and $2.5 \mathrm{~cm}^{3} / \mathrm{rev}$ for direct motion control of the hydraulic cylinder. The pumps are mutually interconnected with the same shaft. Mitsubishi HG-SN152JK speed controlled $1.5 \mathrm{~kW}$ permanent-magnet synchronous motor with a maximal rotational velocity of $2000 \mathrm{rev} / \mathrm{min}$ is used for propelling the pumps. Digital AC servo amplifier MR-JE200A with model reference adaptive control is used as black-box speed controller based on the input voltage signal from the PLC. For the torque transfer from the servo motor to the pumps, a belt transmission is used. The A-side and B-side pumps create the inlet and outlet flow to the cylinder where the movement direction of the cylinder depends on the servo motor rotational direction while the required operating pressure is determined by the payload.

Experimental setup is designed for easy switching between both systems allowing us an exact comparison of obtained experimental results. Wire Micro-Epsilon WDS sensor is used for measuring cylinder position, while 
EVS and HDA sensors from Hydac are used for measuring inlet and outlet flow from the cylinder and pressures inside cylinder chambers. Piston velocity is estimated on-line with an algebraic differentiator defined in [14]. Data logging on a $10 \mathrm{~ms}$ base is implemented on a Mitsubishi PLC along with the proposed control algorithms. The detailed system description is given in [15].

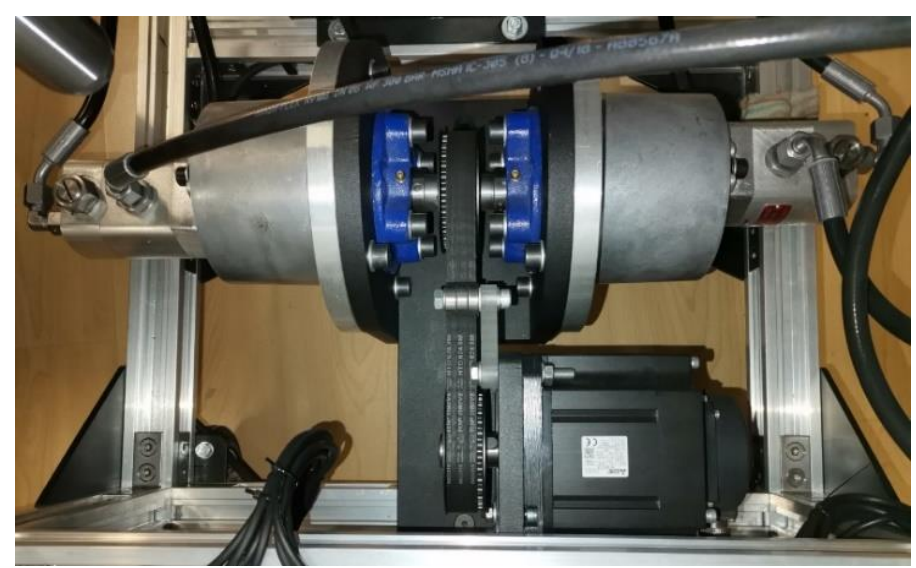

Figure 2: DDH drive

\section{Control algorithms definitions}

The control process of an electrohydraulic system is a tough task due to the nonlinearities that occur in the system. Because of that linear controllers, such as PID regulator, can only achieve good results around the linearization point. Nonlinear control algorithms can easily overcome nonlinearities that occur due to friction phenomena, internal and external leakages and parameter variation. An overview of the control structure for the DDH model is given in fig. 3. Input to the system is desired cylinder position and the output of the system is the cylinder position. The output of the controller is a voltage signal which is sent to a servo drive or proportional valve.

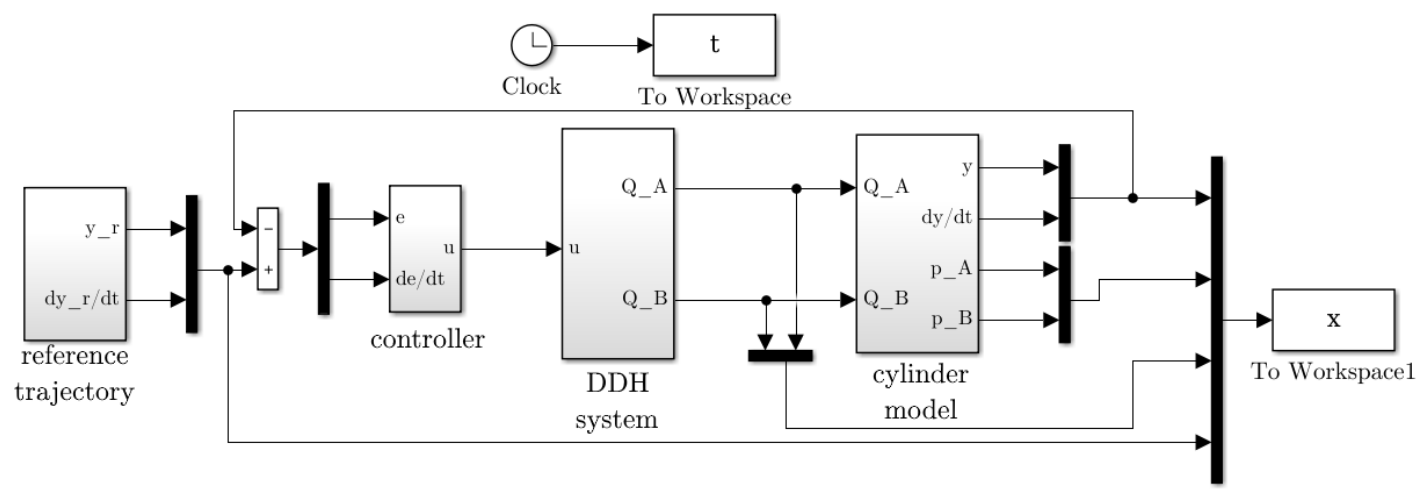

Figure 3: Overview of the control structure of the DDH system

From the defined control structure, the system error is defined as:

$$
e=x_{d}-x_{m}
$$

where $x_{d}$ and $x_{m}$ are desired and measured trajectories respectively.

In control theory, the PID controller is one of the most used regulators in the industry due to its simplicity and satisfying performances in a different kind of process. Today, PID controller is implemented in its discreet form and it can be found in all control devices as a stand-alone controller or as a function block in a PLC. For obtaining the reference results, PID regulator in its general form is used and it's given as:

$$
\mathrm{u}=k_{p}\left(\alpha x_{d}-x_{m}\right)+\int k_{i} e d t+k_{d}\left(\beta \dot{\mathrm{x}}_{d}-\dot{x}_{m}\right)
$$

where $k_{p}, k_{i}$ and $k_{d}$ are proportional, integral and derivate gain respectively, $u$ is the control signal while $\alpha$ and $\beta$ are weighting coefficients for choosing between different structures of a PID controller. With $\alpha=\beta=1$ parallel form of the PID controller is achieved while with $\alpha=\beta=0$ I-PD structure of the controller is obtained. In this paper parallel form of the PID controller is used. The controller parameters are obtained with the Ziegler-Nichols method for the step amplitude of $150 \mathrm{~mm}$. The obtained parameters are used for both test signals and different payloads. 
The sliding mode controller (SMC) is a nonlinear discontinuous robust controller and it is used in presence of the disturbances and system parameter variation. Proposed SMC is given as:

$$
\begin{gathered}
\mathrm{s}=\dot{e}+\lambda \mathrm{e} \\
u=k_{1} s+k_{2} \operatorname{sign}(s)
\end{gathered}
$$

where $s$ is a sliding variable and $k_{l}, k_{2}$ and $\lambda$ are designed parameters and $u$ is the control signal. Variable $k_{l}$ acts on system stability and reference tracking while $k_{2}$ acts on the unknown system dynamics and chattering. Given form of the SMC has second-order dynamics and it only requires system error for calculating the control signal.

\section{System dynamics}

Both systems are subject to a series of test cases in the duration of 60 seconds. The cylinder payload was increased from 0 to $200 \mathrm{~kg}$ with a $20 \mathrm{~kg}$ increment. This approach allows us to test the robustness of the proposed regulators on the different payloads while maintaining the same regulator parameter. All tests are carried out with initial conditions set to zero. The robustness and system dynamics of the proposed regulator are determined through 6 parameters calculated from step and sine wave system response. Conclusions are given for a cycle duration.

\subsection{Sine wave experimental results}

Three parameters are calculated from the results obtained for the sine wave reference trajectory. Amplitude error $\left(A_{e}\right)$ and phase shifting $\left(\rho_{e}\right)$ are calculated from an optimized sine wave signal fitted over recorded data where the objective function which needs to be minimized is Moment of Error Squared (MES) defined as:

$$
M E S=\int t e(t)^{2} d t
$$

Withal, MES criterium is used as a performance metric of a sine wave response. Reference trajectory is given as:

$$
x_{d}(t)=0.15+0.05 \sin (2 \pi \cdot 0.2 t)
$$

System response, error and control signal for the proposed controllers and a fully loaded cylinder are shown in fig. 4. Both controllers showed good tracking capabilities. The maximal absolute tracking error for each controller is about $10 \mathrm{~mm}$. The SMC regulator showed better tracking capabilities than a PID controller and obtained a faster and smoother response.
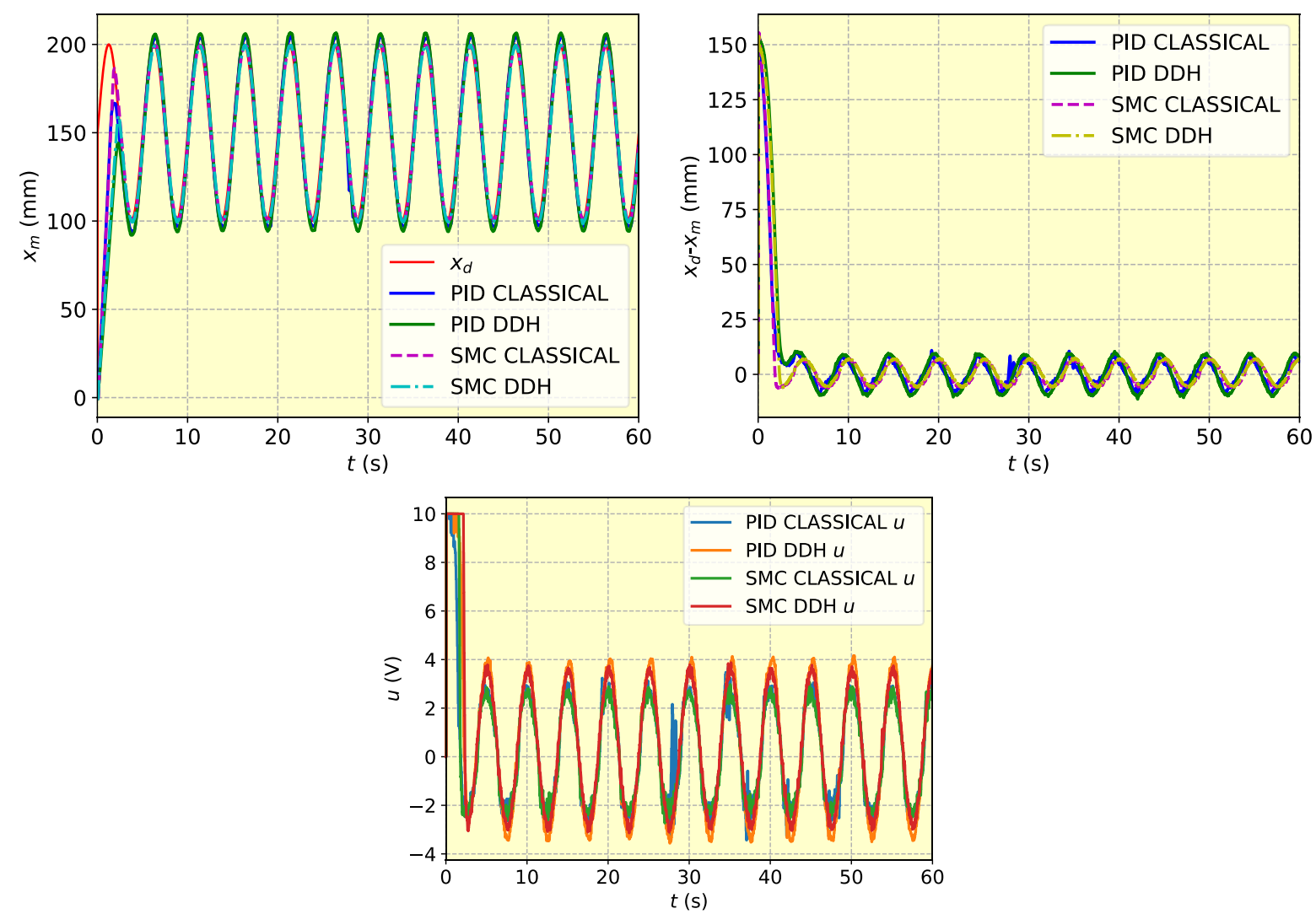

Figure 4 Sine wave experimental results for a fully loaded cylinder 
Decomposition of the system response on amplitude and phase error for the sine wave reference trajectory is shown in fig. 5. The minus sign for the amplitude error means that the amplitude of system response is higher than the desired amplitude. PID controller for the classical system has amplitude error around $-4.4 \mathrm{~mm}$ while for DDH system amplitude error is $-6 \mathrm{~mm}$. SCM regulator showed that both systems have amplitude error around zero while a small deviation occurred for payloads over $80 \mathrm{~kg}$. This deviation is neglectable in comparison to the PID controller. The smallest phase-shifting occurred on the classical system with a PID controller and the largest on a DDH system controller with a PID regulator. For both systems, similar phase-shifting occurred with SMC. The DDH system was accelerated and phase-shifting was lower in comparison to the PID controller while classical systems showed an opposite trend. It's also shown that the PID controller is robust for the small amplitudes of sine wave signal and given payloads. That robustness comes out due to PID parameters obtained for the cylinder middle position $(150 \mathrm{~mm})$ and payload of $100 \mathrm{~kg}$.

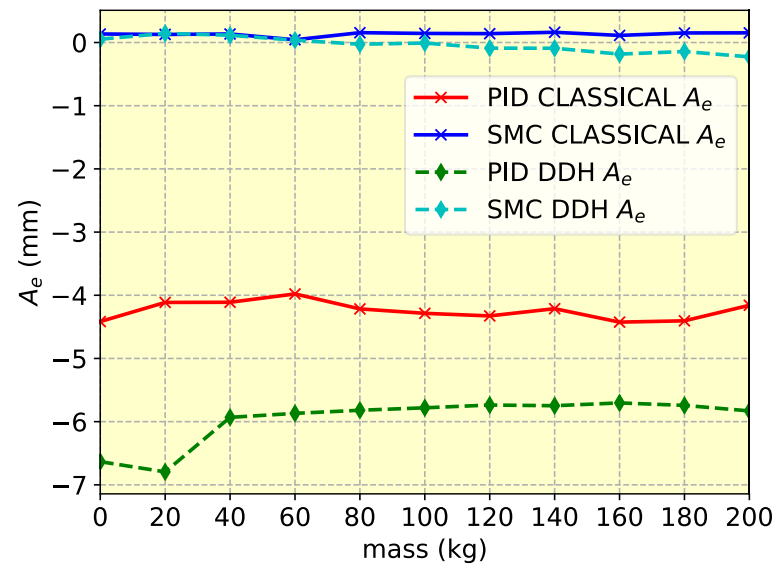

a)

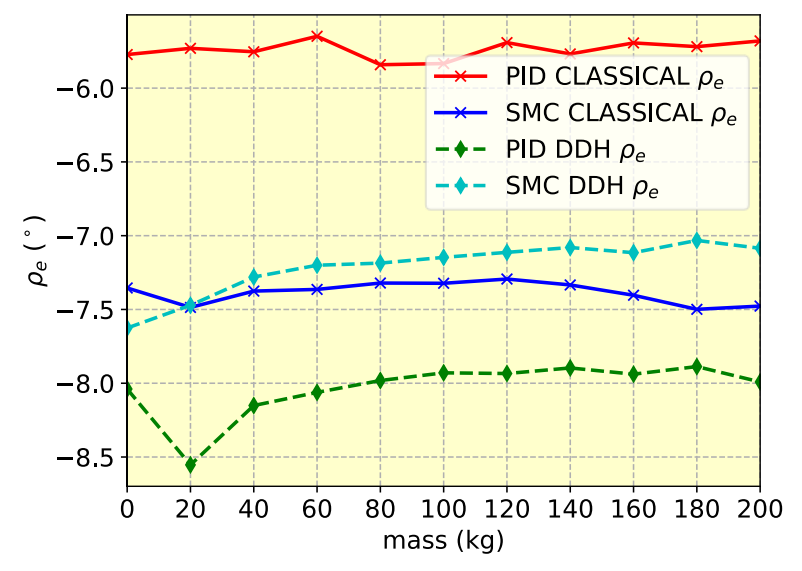

b)

Figure 5 Sine wave decomposition: a) amplitude error, b) phase shifting

As a last performance index for the sine wave results, the $M E S$ criterion (6) is calculated and shown in fig. 6. Both systems obtained small cumulative errors with SMC. For the DDH system cumulative error of SMC is drastically smaller in comparison to the PID controller and it almost overlaps with results obtained with the classical system controlled with a PID controller.

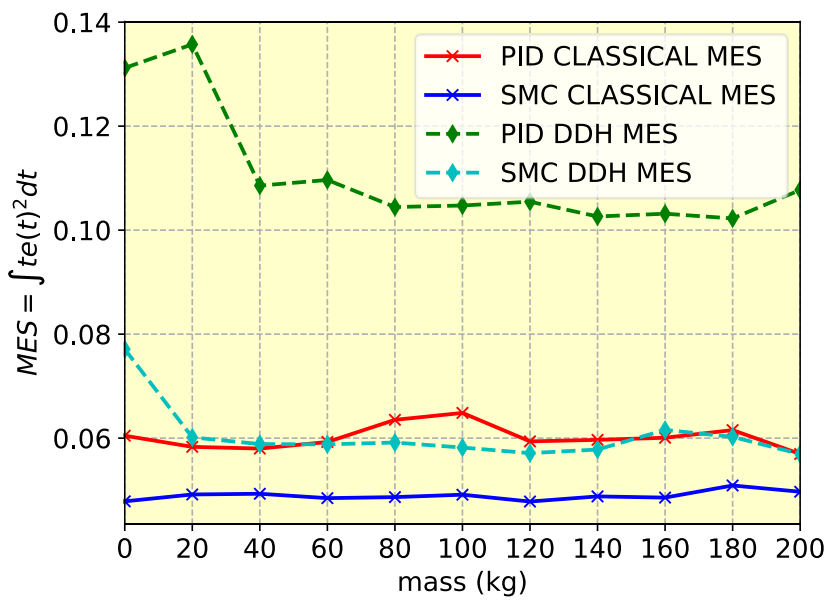

Figure 6 MES criterion for the sine reference signal

\subsection{Step signal experimental results}

Reference test step signal consist of a six different cylinder position set to $280,10,150,22,10$ and $0 \mathrm{~mm}$. The duration of each step signal is set to 10 seconds. From the obtained results, three performance indexes were calculated. Rise time $\left(T_{r}\right)$ is defined as an interval that it takes for the response to rise from $10 \%$ to $90 \%$ of the steady-state value. Settling time $\left(T_{s}\right)$ is the interval that takes for the error to settle between $\pm 1 \mathrm{~mm}$ of a step final value. The last performance index is the Integral of Squared Error (ISE) used as a cumulative measure for the system error and it is defined as: 


$$
I S E=\int e(t)^{2} d t
$$

Step results for a fully loaded cylinder and control signal are shown in fig. 7. Systems controlled with SCM showed a slightly faster response in compression to the PID controller. Due to small differences in response for both controllers detailed analysis is carried out on which conclusion are given.
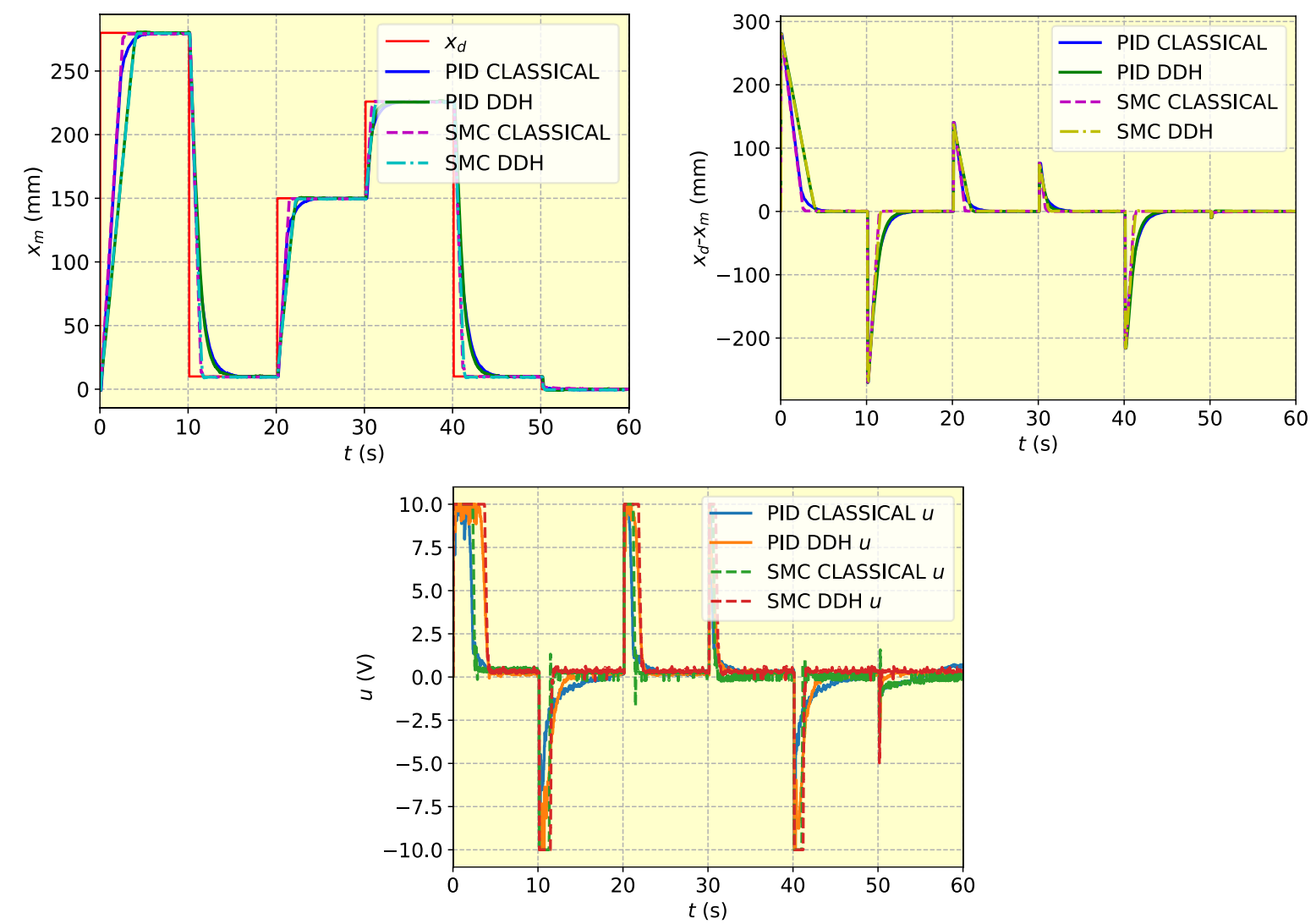

Figure 7 Step signal experimental results for a fully loaded cylinder

Mean values of the rise and settling time for all test cases and every change of the step signal are shown in fig. 8 . From the aspect of rise time, the DDH system controlled with SMC show slightly better performance for lifting movements while faster dynamics is obtained for lowering movements. The SMC for the classical system showed far better results for rise time but results for the small-signal regime aren't satisfying. From the aspect of settling time, the classical system showed the same trend as for rise time. The DDH system controlled with SMC showed slightly higher results in comparison to PID for lifting movements while settling time for lowering movements was lower.

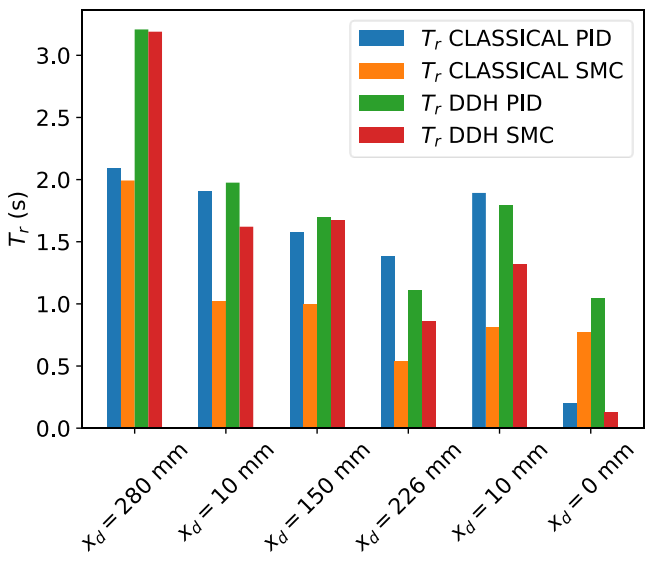

a)

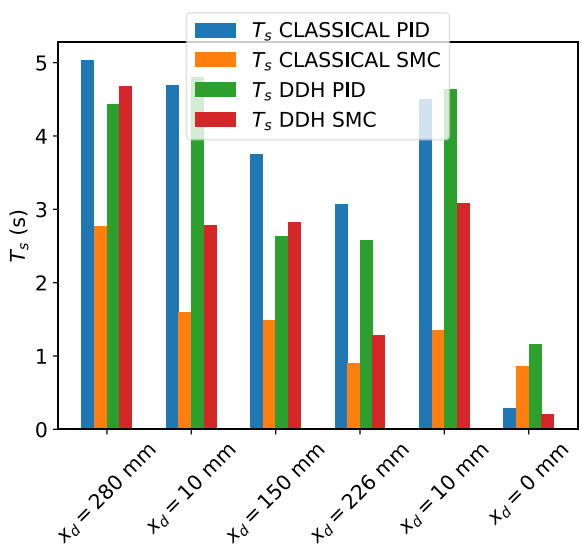

b)

Figure 8 Mean values for: a) rise time, $b$ ) settling time 
The cumulative errors of the systems based on ISE criterium are shown in fig. 9. There is almost no difference between results obtained with SMC and PID controller for the individual system.

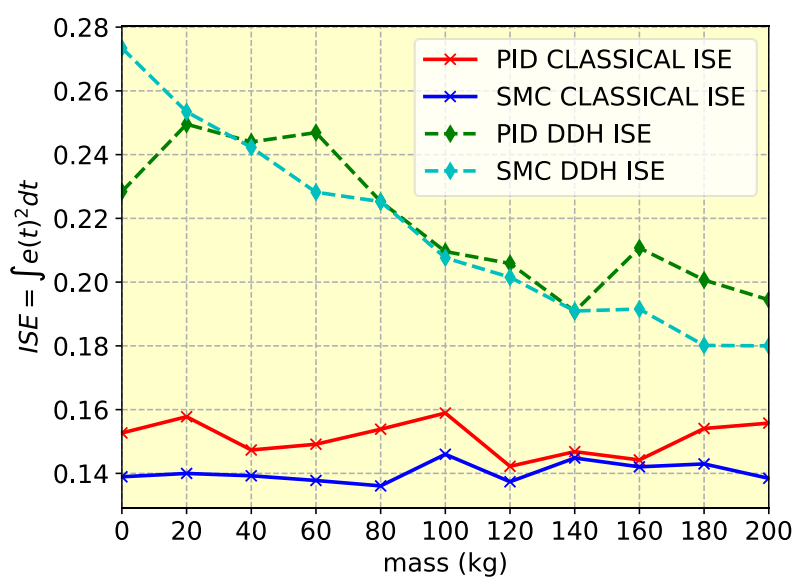

Figure 9 ISE criterion for the step reference signal

\section{Energy efficiency}

The input and output energy of the system is determined based on power flow direction meaning that the negative work done by the hydraulic cylinder is considered as an additional input to the system. Energy regeneration for the DDH system isn't taken into the account due to the impossibility of conducting such experiment but it's possible as presented in [9]. Based on the previous assumption, the input energy of the system is calculated as:

$$
E_{\text {in }}=\int P_{e l} d t+\left.\int\left|P_{c y l}\right|\right|_{P_{c y l}<0} d t
$$

where $P_{e l}$ is input electrical power consumed either by the servo motor or the AC motor and $P_{c y l}$ is a power of the hydraulic cylinder defined as:

$$
P_{c y l}=\left(p_{A} A_{A}-p_{B} A_{B}\right) v_{c y l}
$$

where $A_{A}$ and $A_{B}$ are piston head and rod side areas respectively, $p_{a}$ and $p_{b}$ are pressures inside hydraulic chambers while $v_{c l y}$ is the velocity of the hydraulic cylinder.

The output energy of the system is defined as:

$$
E_{\text {out }}=\left.\int\left|P_{c y l}\right|\right|_{P_{c y l} \geq 0} d t
$$

From (9) and (11) energy efficiency of the proposed system is given as:

$$
\eta=\frac{E_{\text {out }}}{E_{\text {in }}} 100
$$

Input and output energies of the systems for the fully loaded cylinder are shown in tab. 1. Results showed that there is a small difference in input and output energy between different types of controllers for the same system. The main difference occurred between DDH and classical systems due to pressure used for overcoming cylinder payload. The DDH uses variable pressure determined by the payload while the classical system uses higher pressures determined by the pressure set on a safety valve. Safety pressure is set to 60 bars taking into account pressured drop across the proportional valve (10 bars) and dynamic payload forces. Pressure comparison is shown in fig. 10 . 
Table 1 Input and output energies of the systems for the fully loaded cylinder

\begin{tabular}{|c|c|c|c|c|c|c|c|}
\cline { 3 - 8 } \multicolumn{2}{c|}{} & \multicolumn{3}{c|}{ PID } & \multicolumn{3}{c|}{ SMC } \\
\cline { 2 - 8 } \multicolumn{2}{c|}{} & $E_{\text {in }}(\mathrm{kJ})$ & $E_{\text {out }}(\mathrm{kJ})$ & $\eta(\%)$ & $E_{\text {in }}(\mathrm{kJ})$ & $E_{\text {out }}(\mathrm{kJ})$ & $\eta(\%)$ \\
\hline $\begin{array}{c}\text { DDH } \\
\text { system }\end{array}$ & sinus & 10.55 & 3.08 & 29.17 & 10.06 & 2.79 & 27.77 \\
\cline { 2 - 8 } & step & 8.38 & 1.09 & 13.05 & 9.97 & 1.10 & 11.07 \\
\hline \multirow{2}{*}{$\begin{array}{c}\text { classical } \\
\text { system }\end{array}$} & sinus & 77.34 & 2.97 & 3.84 & 78.42 & 2.79 & 3.55 \\
\cline { 2 - 8 } & step & 76.08 & 1.08 & 1.42 & 74.91 & 1.10 & 1.46 \\
\hline
\end{tabular}

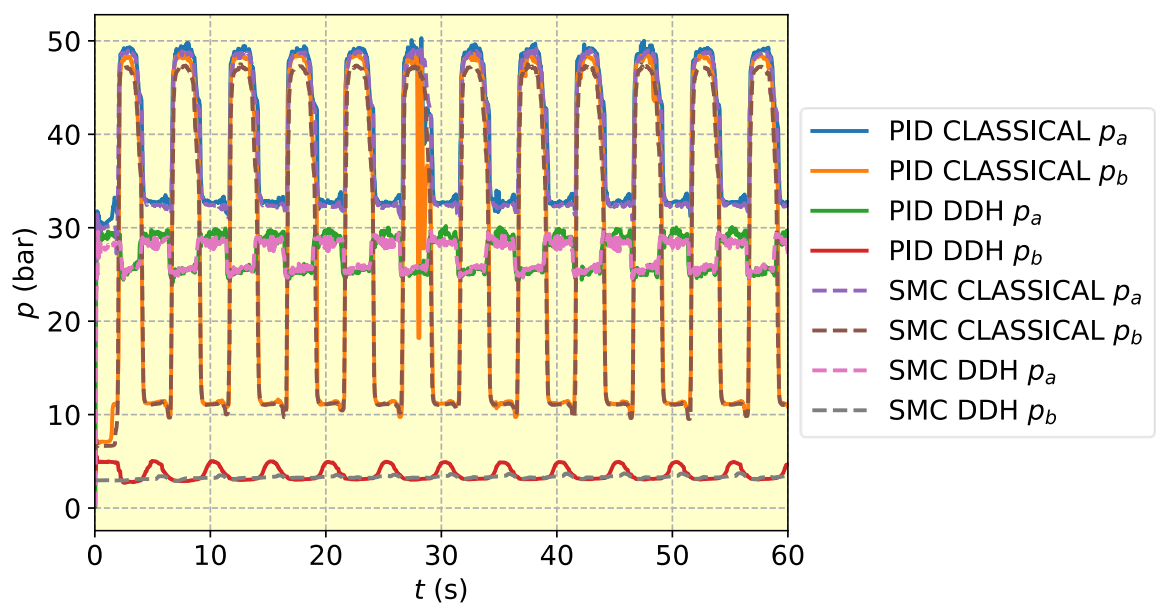

Figure 10 Pressures inside cylinder chamber

Energy efficiency calculated from (12) for a given test signal is shown in fig. 11. Results showed that the energy efficiency of a classical system is almost identical for both controllers. The DDH system controlled with a PID controller has slightly better energy efficiency in comparison to SMC but both controllers have superior efficiency in comparison to the classical electrohydraulic system.

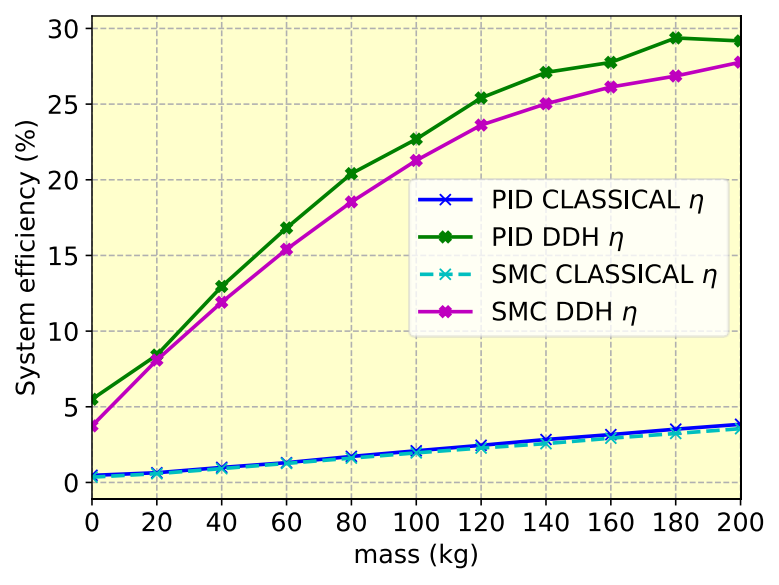

a)

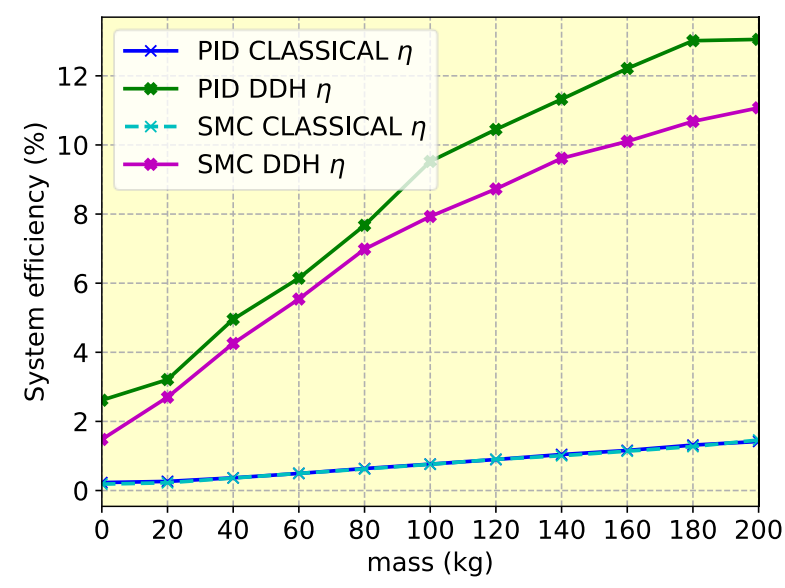

b)

Figure 11 Energy efficiency for: a) sine wave reference signal, b) step reference signal

\section{Conclusion}

The DDH system controlled with SMC obtained faster dynamics in comparison to the PID controller. Such faster dynamics showed an impact on the energy efficiency which is slightly lower than one calculated from results obtained with the PID controller giving us an acceptable trade-off. Classical system controlled with SMC showed slight improvements in system dynamics and almost no impact on energy efficiency in comparison to PID controller. All overall, the SMC controller for the DDH system showed superior results in comparison to a PID controlled classical system. Furthermore, the additional energy efficiency of the DDH system can be achieved if energy regeneration is utilized for lowering movements. The system dynamics will be additionally investigated in 
future work with the integrator backstepping controller. Such controller should achieve excellent tracking capabilities for sine wave reference trajectory and better results for the rise and settling time for step reference. Energy efficiency will be slightly lower, but still acceptable. Additionally, a detailed energy efficiency model will be developed. The proposed model will include hydro-mechanical, hydraulic and electrical losses.

\section{Acknowledgments}

It is gratefully acknowledged that this research has been supported by the European Regional Development Fund under the grant KK.01.1.1.04.0010 (HiSkid). The authors would like to acknowledge and thank Mr. M. Cavka from Hidromehanika d.o.o, Mr. H. Zidar from ABC maziva d.o.o. and Mrs. O. Tomić from HANSA-FLEX Croatia d.o.o. who greatly contributed to the development of this laboratory model and provided their assistance in the hardware setup.

\section{Nomenclature}

\begin{tabular}{|c|c|c|}
\hline Designation & Denotation & Unit \\
\hline$A_{A}$ & piston head area & $\mathrm{m}^{2}$ \\
\hline$A_{b}$ & piston rod-side area & $\mathrm{m}^{2}$ \\
\hline$e$ & error between desired and measured trajectory & $\mathrm{mm}$ \\
\hline$E_{\text {in }}$ & input energy of the system & $\mathrm{J}$ \\
\hline$E_{\text {out }}$ & output energy of the system & $\mathrm{J}$ \\
\hline$k_{1}$ & SMC design parameter & - \\
\hline$k_{2}$ & SMC design parameter & - \\
\hline$k_{d}$ & PID controller derivate gain & - \\
\hline$k_{i}$ & PID controller integral gain & - \\
\hline$k_{p}$ & PID controller proportional gain & - \\
\hline$p_{a}$ & pressure on piston head side & $\mathrm{Pa}$ \\
\hline$p_{a}$ & pressure on piston rod side & $\mathrm{Pa}$ \\
\hline$P_{c y l}$ & hydraulic cylinder power & $\mathrm{W}$ \\
\hline$P_{e l}$ & input electrical power to the system & $\mathrm{W}$ \\
\hline$s$ & sliding variable & - \\
\hline$T_{r}$ & rising time & $\mathrm{s}$ \\
\hline$T_{s}$ & settling time & $\mathrm{s}$ \\
\hline$u$ & control signal & $\mathrm{V}$ \\
\hline$U_{e f f}$ & effective voltage of AC network & $\mathrm{V}$ \\
\hline$v_{c y l}$ & piston velocity & $\mathrm{m} / \mathrm{s}$ \\
\hline$x_{d}$ & desired trajectory & $\mathrm{mm}$ \\
\hline$x_{m}$ & measured trajectory & $\mathrm{mm}$ \\
\hline$\alpha$ & weighting coefficients & - \\
\hline$\beta$ & weighting coefficients & - \\
\hline$\eta$ & system efficiency & $\%$ \\
\hline$\lambda$ & SMC design parameter & - \\
\hline
\end{tabular}




\section{References}

[1] M. Ivantysynova, "Innovations in pump design-what are future directions?," in Proceedings of the JFPS International Symposium on Fluid Power, 2008.

[2] J. Siebert, M. Wydra and M. Geimer, "Efficiency Improved Load Sensing System—Reduction of System Inherent Pressure Losses,” Energies, vol. 10, p. 941, 72017.

[3] J.-m. Zheng, S.-d. Zhao and S.-g. Wei, "Adaptive Fuzzy PID Control for Switched Reluctance Motor Direct Drive Servo Hydraulic Press," in 2009 International Conference on Measuring Technology and Mechatronics Automation, 2009.

[4] O. D. A. Hiroyuki, K. OHTSU, S. A. T. O. Hiroshi and K. KANEHIRO, "Designing advancedrudder roll stabilization system - Using High Power with Small SizeHydraulic System," in Proceedings of the JFPS International Symposium on Fluid Power, 2008.

[5] S. Kuchler, O. Sawodny, K. Schneider and K. Langer, "Vibration damping for a hydraulic driven luffing cylinder at a boom crane using feedforward control," in 2009 IEEE/ASME International Conference on Advanced Intelligent Mechatronics, 2009.

[6] T. Minav, C. Bonato, P. Sainio and M. Pietola, "Direct Driven Hydraulic Drive," in The 9th International Fluid Power Conference, 9. IFK, March 24-26, 2014, Aachen, Germany, 2014.

[7] T. A. Minav, J. E. Heikkinen and M. Pietola, "Electric-driven Zonal Hydraulics in Non-Road Mobile Machinery," in New Applications of Electric Drives, InTech, 2015.

[8] T. A. Minav, P. Sainio and M. Pietola, "Direct-Driven Hydraulic Drive Without Conventional Oil Tank," in ASME/BATH 2014 Symposium on Fluid Power and Motion Control, 2014.

[9] T. A. Minav, C. Bonato, P. Sainio and M. Pietola, "Efficiency of direct driven hydraulic drive for non-road mobile working machines," in 2014 International Conference on Electrical Machines (ICEM), 2014.

[10] A. Niraula, S. Zhang, T. Minav and M. Pietola, "Effect of Zonal Hydraulics on Energy Consumption and Boom Structure of a Micro-Excavator,” Energies, vol. 11, p. 2088, 82018.

[11] S. Zhang, T. Minav and M. Pietola, "Decentralized Hydraulics for Micro Excavator," in Proceedings of 15:th Scandinavian International Conference on Fluid Power, 15th Scandinavian International Conference on Fluid Power, Fluid Power in the Digital Age, SICFP'17, June 7-9 2017 - Linköping, Sweden, 2017.

[12] T. Minav, J. Heikkinen, T. Schimmel and M. Pietola, "Direct Driven Hydraulic Drive: Effect of Oil on Efficiency in Sub-Zero Conditions," Energies, vol. 12, p. 219, 12019.

[13] T. Minav, M. Pietola, D. M. Filatov, A. V. Devyatkin and J. Heikkinen, "Fuzzy control of direct-driven hydraulic drive without conventional oil tank," in 2017 XX IEEE International Conference on Soft Computing and Measurements (SCM), 2017.

[14] J. Kasac, D. Majetic and D. Brezak, "An algebraic approach to on-line signal denoising and derivatives estimation,” Journal of the Franklin Institute, vol. 355, p. 7799-7825, 102018.

[15] J. Benić and Ž. Šitum, "Position Controller for Direct Driven Electro-Hydraulic System," in International conference Fluid Power 2019: Conference Proceedings, 2019. 